\title{
Editorial
}

\section{Scientific Electronic Library Online (SciELO) y Oncología}

\section{Introducción}

Durante más de medio siglo las publicaciones españolas se han encontrado con enormes dificultades a la hora de intentar penetrar en los principales núcleos del conocimiento de la comunidad científica internacional.

Entre las múltiples causas que han influido en esta situación, se encuentra el escaso peso internacional que suponía la ciencia española durante el periodo de estructuración de los primeros núcleos editoriales, y que desde entonces hasta la actualidad, se han convertido en los principales promotores del conocimiento científico.

Si bien este hecho pudo suponer un peso importante en su momento, en la actualidad no constituye, ni el único ni el principal de los problemas que mantienen esta situación. De hecho, mientras la comunidad científica española se ha recuperado de forma espectacular hasta ocupar en la actualidad un honroso papel en el ranking mundial, la visibilidad internacional de las publicaciones editadas en España, no se ha visto recompensada en la misma proporción. Así, se ha consolidado la representatividad de la ciencia española en el marco internacional, lo que en paralelo se ha traducido en un incremento de la presencia de su producción en el escenario de elite científica. Por el contrario, la escasez de iniciativas orientadas a crear herramientas que facilitasen el acceso a fuentes de información y la difusión de "otra parte" de la producción científica, también de calidad, pero concentrada en ediciones españolas, ha supuesto una dificultad añadida a la hora de mantener la situación de desequilibrio.

La presencia de Internet y la aparición de redes del conocimiento en el entorno de nuevas tecnologías de la información, así como determinadas iniciativas para incrementar la visibilidad y presencia internacional de publicaciones de calidad contrastada que permanecían escasamente visibles, han permitido acceder al escaparate universal a un considerable número de publicaciones españolas que cumplen cánones preestablecidos de calidad internacional.

\section{Oncología en la Red SciELO, una clara apuesta por su difusión}

Un claro ejemplo de publicación española con un nivel de calidad suficiente dentro de los cánones internacionales lo podemos encontrar en la revista Oncología, que se encuentra referenciada en más de seis bases de datos bibliográficas internacionales, entre las que podemos citar Biological Abstracts, Chemical Abstracts, CAB Abstracts, CAB Health y EMBASE/The Excepta Medica Database, entre otras, además de en IME (Indice Médico Español) y en el Índice Bibliográfico Español de Ciencias de la Salud (IBECS) (http://ibecs.isciii.es)

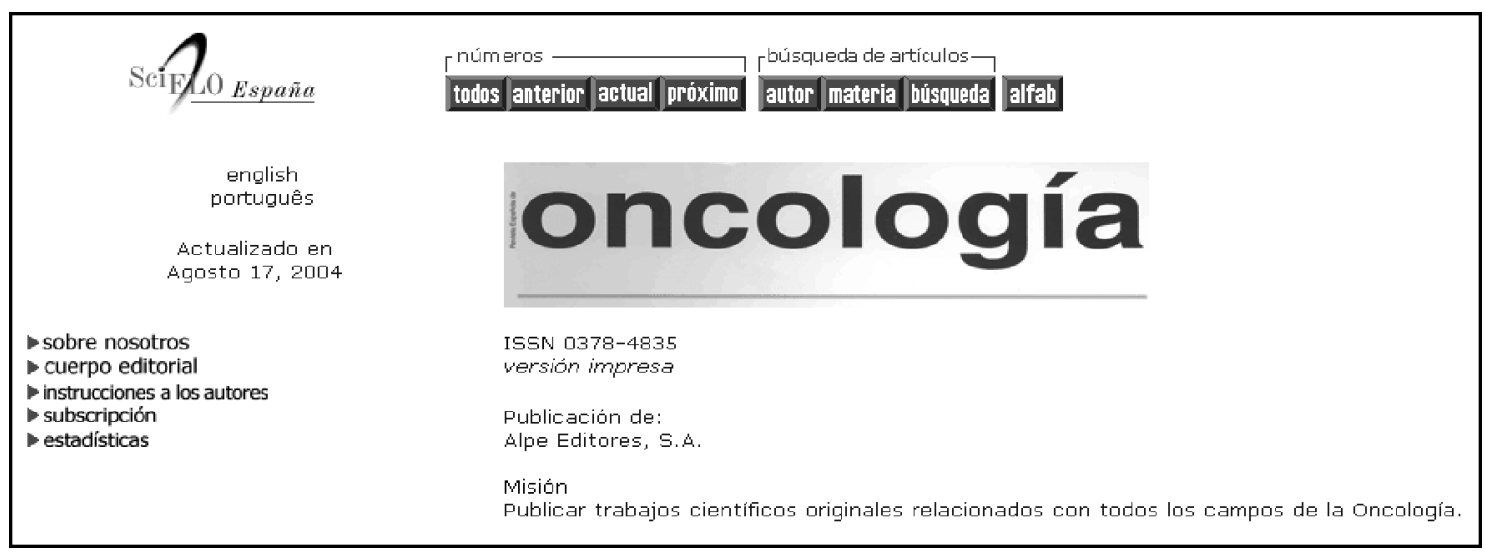

Fig. 1. Acceso a la revista Oncología desde SciELO España 


\section{J. Veiga de Cabo}

Esta última, utiliza los mismos tesauros (MeSH y DeCS) o motores de búsqueda con los que operan las bases de datos internacionales Medline y Lilacs, por lo que es compatible con ellas. El hecho de que Oncología se encuentre referenciada en IBECS supone que ha superado los criterios de calidad estandarizados para bases de datos internacionales, además de su accesibilidad desde la Red de Bibliotecas Virtual en Salud de la Organización Panamericana de la Salud de la Organización Mundial de la Salud (OPS/OMS), lo que supone una mayor difusión de los contenidos de la revista por encontrarse en un escaparate internacional de acceso a fuentes de información (250 visitas diarias de media en BVS España) (http://bvs.isciii.es).

Pero, a partir de enero de 2004, Oncología se ha integrado en SciELO España (Fig. 1) y la Red SciELO (Scientific Electronic Library Online), lo que supone el acceder al texto completo de la revista a través de una de las principales Redes internacionales con mayores cotas de prestigio alcanzada en los últimos años.

\section{¿Qué es SciELO?}

\section{Red SciELO}

Scientific Electronic Library Online (Red SciELO) (http://www.scielo.org), es un proyecto internacional liderado por el Centro Latinoamericano y del Caribe de Información en Ciencias de la Salud (BIREME) dependiente de la Organización Panamericana de la Salud - Organización Mundial de la Salud (OPS/OMS), en el que intervienen diferentes países. En la actualidad, la Red SciELO se encuentra integrada por SciELO Brasil, Chile, Cuba, España (http://scielo.isciii.es) y un portal temático (SciELO Salud Pública). Países como Colombia, Costa Rica México, Portugal, Perú y Venezuela se encuentran en fase piloto (Fig. 2).

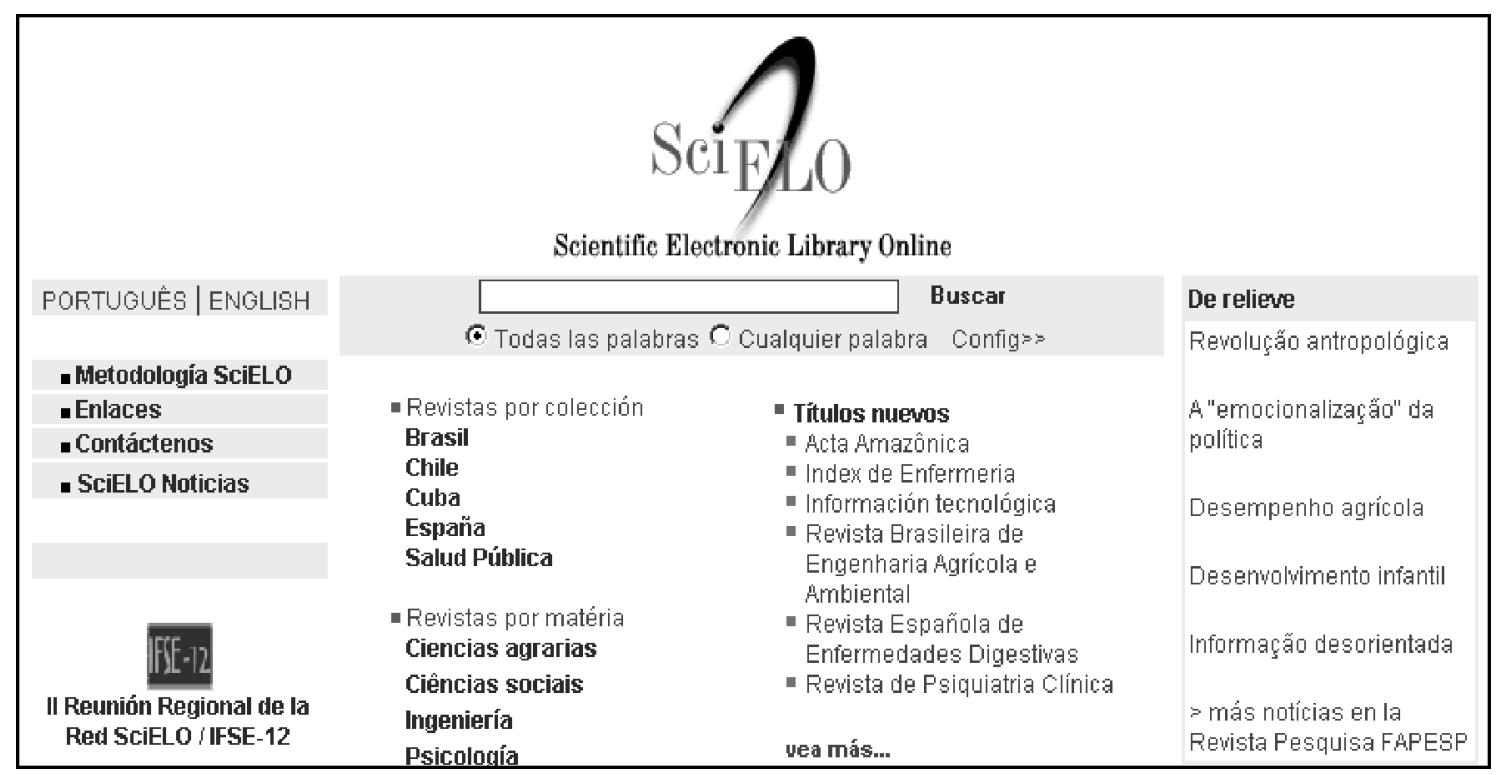

Fig. 2. Portal Red SciELO

La metodología SciELO se basa en un sistema de acceso abierto (open acces) que utiliza criterios internacionales de calidad previamente establecidos como medida de control de contenidos. Permite acceder a través de Internet al texto completo de unas 300 publicaciones científicas de calidad certificada, editadas en cada uno de los países que la integran. De ellas, aproximadamente la mitad se corresponden con diferentes especialidades del área de Ciencias de la Salud (Tabla I). 
TABLA I

Número de revistas por áreas temáticas y países

\begin{tabular}{lcccc}
\hline País & Año inicio & C. Salud & Otras áreas & Total \\
\hline Brasil & 1998 & 47 & 108 & 155 \\
Chile & 1999 & 14 & 32 & 46 \\
Cuba & 2001 & 17 & - & 17 \\
España & 2001 & 20 & - & 20 \\
Costa Rica * & 2000 & 8 & 1 & 9 \\
México $^{*}$ & 2003 & 10 & - & 10 \\
Venezuela $^{*}$ & 2000 & 11 & 27 & 38 \\
Colombia $^{*}$ & 2004 & 5 & - & 5 \\
Perú & 2004 & 10 & 12 & 312 \\
Total & & 142 & 170 & \\
\hline
\end{tabular}

${ }^{*}$ Colecciones en desarrollo

El diseño utilizado por SciELO permite una interacción cómoda entre el usuario y el sistema, al utilizar una metodología común de los espacios que integran la Red. Esto permite que la información aparezca estructurada siempre de la misma forma y facilita mucho la navegación.

El modelo SciELO integra diferentes componentes que permiten, 1) acceso al texto (HTML y/o pdf) (Fig. 3) así como poder recuperar información a través de una base de datos bibliográfica que permite buscar por autor, palabras del título, materia, año de publicación y afiliación, de forma individual o combinando campos (Fig. 4), 2) acceder desde la bibliografía de un artículo a los registros bibliográficos de resúmenes y contenidos de bases de datos bibliográficas como MedLine, LILACS, IBECS cuando éstos se encuentran referenciados en ellas, así como al texto completo si el artículo ha sido editado anteriormente en SciELO (Fig. 5), 3) obtener de forma directa la referencia de cómo citar el artículo mediante normas ISO (formato impreso y electrónico) (Fig. 6), 4) acceder a la Red internacional de investigadores SciENTI que facilita información complementaria sobre líneas de investigación y publicaciones de los autores de los artículos (Fig. 6) y 5) acceder a un módulo de utilización, uso e impacto de las publicaciones periódicas integradas en la red SciELO.

\section{Oncología (Barcelona)}

ISSN 0379-4835 versión imoresa

La Oncoología Radioterápica en el Tercer Milenióo. Päte II

Tabla de conterido

Oncología (Barci) v.27 n.7 Madrid jul, 20104

- Cáncer de Próstata

- Experiencia con radioterapia externa y sobreimpresión con braquiterapia HDR y LDR en el tratamiento del cáncer de próstata

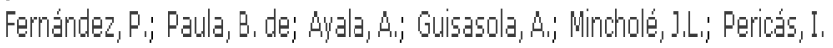

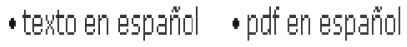

Fig. 3. Acceso al texto completo de los artículos en formato html y pdf 


\section{J. Veiga de Cabo}

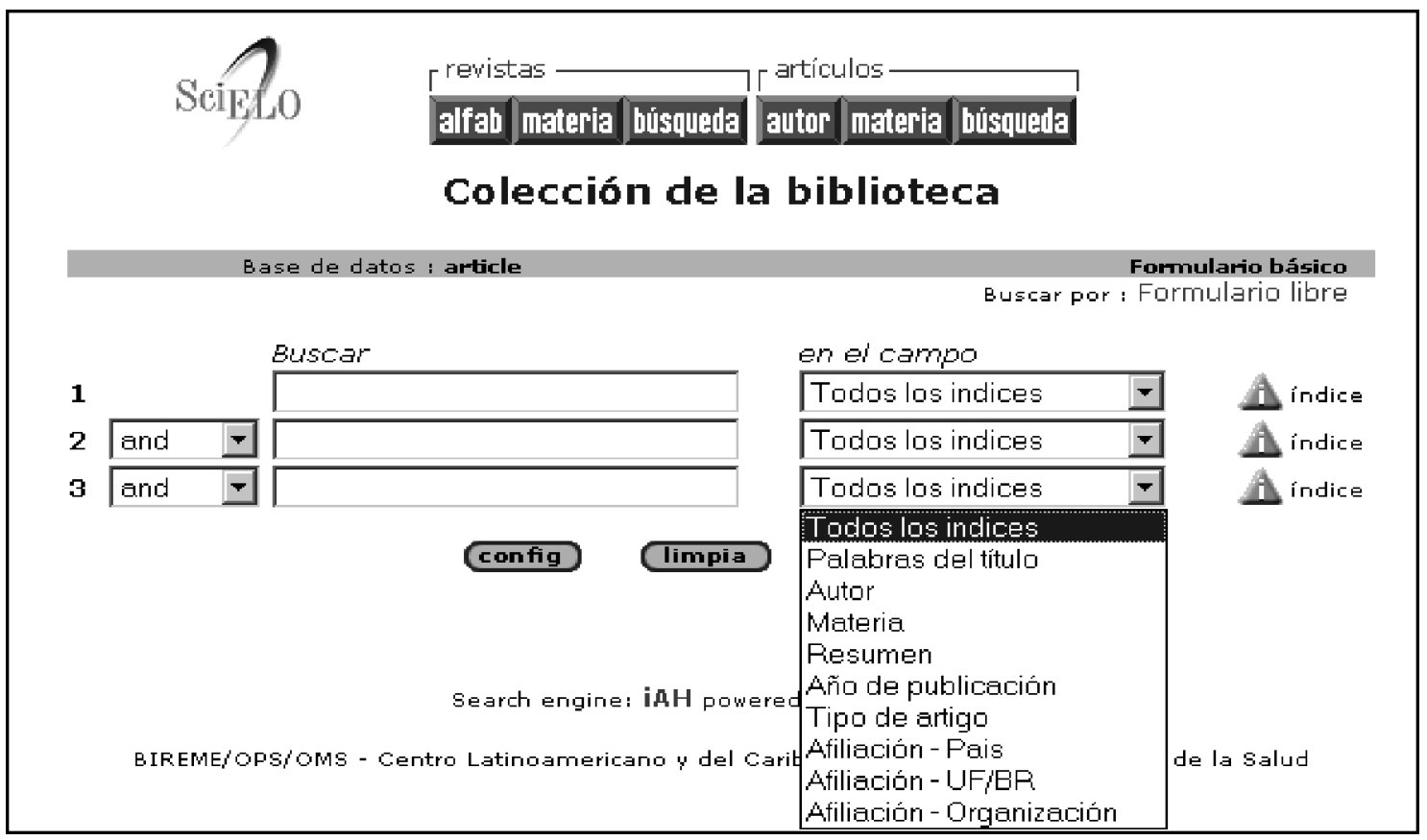

Fig. 4. Búsqueda por campos utilizando el formulario básico

7. Costa-Macedo LM, Machado-Silva JR, Rodrigues-Slva R, Olveira LM, Vianna MSR. Enteroparasitoses em pré-escolares de comunidades favelizadas da cidade do Rio de Janeiro, Brasil. Cadernos de Saúde Pública 14:851-855, 1998.

[ Medline ] [Lilacs ] [

Fig. 5. Enlace con otras bases de datos

\section{Q. Curriculum ScienTI \\ Como citar este artícula}

Fig. 6. Acceso a la red ScienTl y normas ISO de citación

\section{SciELO-España}

A partir de 1999, la Biblioteca Nacional de Ciencias de la Salud (BNCS) del Instituto de Salud Carlos III (ISCIII) se incorpora a la Red SciELO en calidad de Centro coordinador en España en la Red e iniciándose el desarrollo de SciELO España (Fig. 7).

En el año 2001 comienza a funcionar SciELO-España en fase piloto con 4 publicaciones periódicas y coincidiendo con el VI Congreso Regional de Información en Ciencias de la Salud, celebrado en Puebla, México, en mayo 2003, se anunció oficialmente la certificación e integración de SciELO España (http://scielo.isciii.es) en la Red SciE- 
Oncología, 2004; 27 (9):519-524

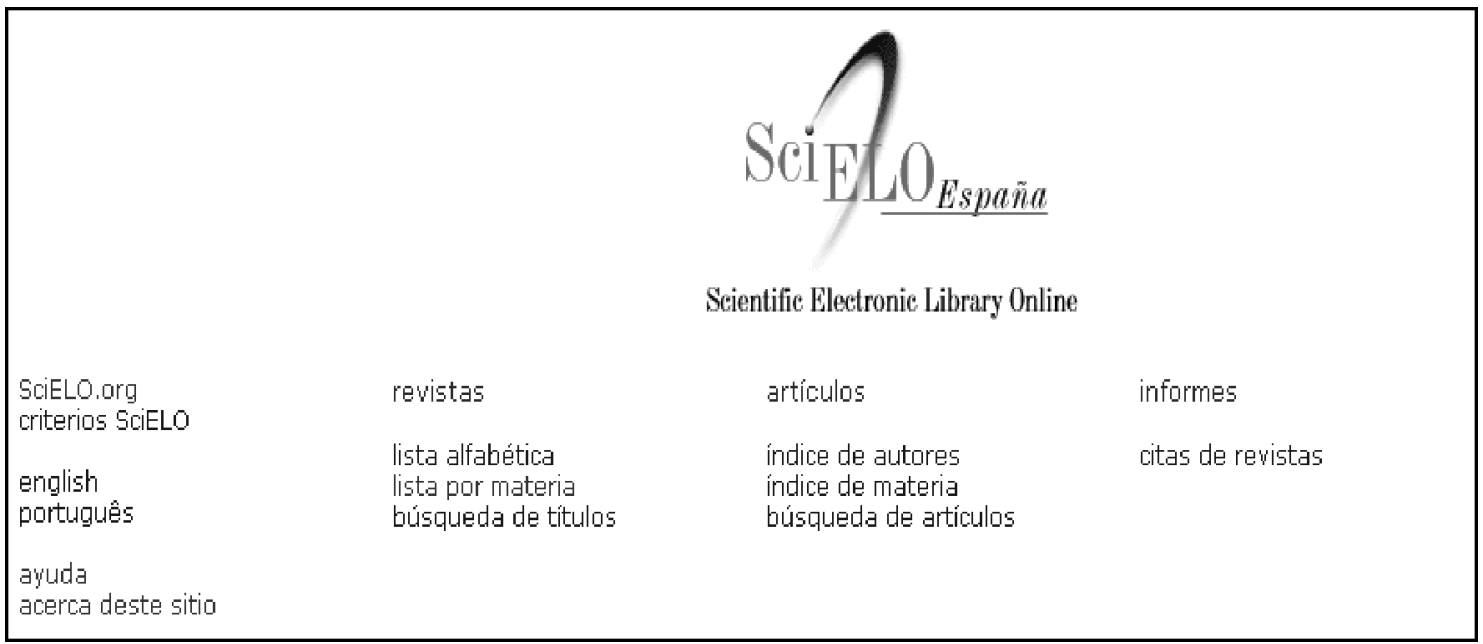

Fig. 7. Portal SciELO España

TABLA II

Distribución de títulos de publicaciones periódicas incluidas en SciELO España por áreas temáticas

\begin{tabular}{|c|c|}
\hline Área & Publicaciones Periódicas \\
\hline Medicina & $\begin{array}{l}\text { Medifam } \\
\text { Anales de Medicina Interna } \\
\text { Revista de Diagnóstico Biológico } \\
\text { Revista Española de Enfermedades Digestivas }\end{array}$ \\
\hline Nutrición & Nutrición Hospitalaria \\
\hline Medicina Forense & Cuadernos de Medicina Forense \\
\hline Odontología & $\begin{array}{l}\text { RCOE } \\
\text { Revista de Cirugía Oral y Maxilofacial } \\
\text { Medicina Oral }\end{array}$ \\
\hline Oncología & Oncología \\
\hline Urología & Actas Urológicas Españolas \\
\hline Salud Pública & $\begin{array}{l}\text { Revista Española de Salud Pública } \\
\text { Gaceta Sanitaria } \\
\text { Anales del Sistema Sanitario de Navarro }\end{array}$ \\
\hline Psiquiatría & The European Journal of Psychiatry (edición en español) \\
\hline Educación Sanitaria & Educación Médica \\
\hline Enfermería & Index de Enfermería \\
\hline Anestesiología & Revista de la Sociedad Española del Dolor \\
\hline Microbiología & International Microbiology \\
\hline Oftalmología & Archivos de la Sociedad Española de Oftalmología \\
\hline
\end{tabular}

LO, pasando a formar parte como miembro del Comité Internacional de la Red. En la actualidad cuenta con un total de 20 publicaciones de 13 áreas de ciencias de la salud (Tabla II). 


\section{J. Veiga de Cabo}

\section{Impacto internacional de la Red SciELO}

En el corto periodo de tiempo que lleva de existencia, la Red SciELO ha logrado abrirse hueco entre los más prestigiosos sistemas de acceso abierto, recibiendo en la actualidad cerca de 2 millones de visitas mensuales. El número de visitantes se han incrementado casi en un $80 \%$ desde que se encuentra indizado en Google.

Otro dato importante de la enorme expansión alcanzada por SciELO durante los últimos años es el hecho de encontrarse enlazado permanentemente a PubMed, WoS, DOAJ, OCLC, WordCat, LoC, LILACS, MedLine, IBECS y BioMed Central, entre otros.

Los resultados de un estudio realizado por la Universidad de Oxford y publicado en Nature en el año 2002, ofrecen datos que ayudan a comprender el potencial de SciELO. Se seleccionaron cinco revistas indizadas por ISI durante más de cinco años y que se mantuvieron accesibles desde SciELO durante un periodo superior a dos años, los factores de impacto de dichas revistas se habían incrementado en más del doble (132.7\% p<0.02, Test de Wilcoxon) desde su inclusión en SciELO'.

Por último, en la II Reunión Regional de la Red SciELO celebrada en el mes de octubre en Mérida, México, se dio a conocer otro dato igualmente indicativo de la difusión que pueden alcanzar los contenidos de SciELO. Lewis Joel Greene, director de la revista Brazilian Journal of Medical and Biological Research, indizada en Medline y en ISI, destacó el aumento del 100 \% del número de citas y del factor de impacto en ISI desde su ingreso en SciELO.

Aunque es difícil predecir para cada revista el alcance en cuanto a difusión y beneficios que pueda obtener por el hecho de encontrarse en SciELO, los datos de los que disponemos hacen sospechar que Oncología se podrá favorecer en igual medida que otras revistas anteriormente incorporadas en la Red SciELO.

Por último, en nombre de la Biblioteca Nacional de Ciencias de Ciencias de la Salud del Instituto de Salud Carlos III, quisiera dar la bienvenida a Oncología y agradecer a los responsables de la revista la confianza depositada, esperando que pronto puedan evidenciarse los éxitos que todos esperamos que se obtengan.

Jorge Veiga de Cabo

Director de la Biblioteca Nacional de Ciencias de la Salud Instituto de Salud Carlos III

' Alonso WJ, Fernández-Juricic E. Regional network raises profile of local journals [carta]. Nature, 2002;415:471-472. 